Journal of Mathematics and Statistics 5 (3):234-240, 2009

ISSN 1549-3644

(C) 2009 Science Publications

\title{
Modeling and Forecasting Volatility of the Malaysian Stock Markets
}

\author{
Ahmed Shamiri and Zaidi Isa \\ School of Mathematical Sciences, University Kebangsaan Malaysia, \\ 43600 UKM, Bangi, Malaysia
}

\begin{abstract}
Problem statement: One of the main purposes of modeling variance is forecasting, which is crucial in many areas of finance. Despite the burgeoning interest in and evaluation of volatility forecasts, a clear consensus on witch volatility model/or distribution specification to use has not yet been reached. Therefore, the out of-sample forecasting ability should be a natural model selection criterion for volatility models. Approach: In this study, we used high-frequency to facilitate meaningful comparison of volatility forecast models. We compared the performance of symmetric $\mathrm{GARCH}$, asymmetric EGARCH and non leaner asymmetric NAGARCH models with six error distributions (normal, skew normal, student-t, skew student-t, generalized error distribution and normal inverse Gaussian). Results: The results suggested that allowing for a heavy-tailed error distribution leads to significant improvements in variance forecasts compared to using normal distribution. It was also found that allowing for skewness in the higher moments of the distribution did not further improve forecasts. Conclusion: Successful volatility model forecast depended much more heavily on the choice of error distribution than the choice of GARCH models.
\end{abstract}

Key words: GARCH-models, asymmetry, stock market indices and volatility modeling

\section{INTRODUCTION}

Traditional regression tools have shown their limitation in the modeling of high-frequency (weekly, daily or intra-daily) data. Assuming that only the mean response could be changing with covariates while the variance remains constant over time often revealed to be an unrealistic assumption in practice. This fact is particularly obvious in series of financial data where clusters of volatility can be detected visually. During the last few decades we have seen a multitude of different suggestions for how to model the second moment, often referred to as volatility, of financial returns. Indeed, it is now widely accepted that high frequency financial returns are heteroskedastic. Among the models that have proven the most successful are the Auto-Regressive Conditional Heteroskedasticity $(\mathrm{ARCH})$ family of models introduced by Engle ${ }^{[4]}$ and the models of Stochastic Variance (SV) pioneered by Taylor $^{[14]}$. One of the main purposes of modeling variance is forecasting, which is crucial in many areas of finance such as option pricing, value at risk applications and portfolio selection. Therefore, the out of-sample forecasting ability should be a natural model selection criterion for volatility models. The vast majority of variance forecasting articles have used squared daily returns as the proxy for ex post variance. This is, as shown by Andersen and Bollerslev ${ }^{[2]}$, an unbiased but exceedingly noisy estimator.

While the literature that examines competing variance models is abundant, very little work has been done comparing different distribution assumptions, with the noticeable exceptions of Anders ${ }^{[1]}$ and Shamiri ${ }^{[10,11]}$. However, none of these papers has explicitly focused on evaluating asymmetric GARCH models forecast using different error distributions. The previous studies paid more attention on the symmetric GARCH model, while in this study we focus on both symmetric and asymmetric volatility models as well as symmetric and asymmetric distributions. Unfortunately, ARCH models often do not fully capture the thick tails property of high frequency financial time series. However, another striking characteristic of high-frequency financial returns is that they are often characterized by fat-tailed distribution. In fact, the kurtosis of most asset returns is higher than three, which means that extreme values are observed more frequently that for the normal distribution. While the high kurtosis of the returns is a well-established fact, the situation is much more obscure with regard to the symmetry of the distribution.

Corresponding Author: Ahmed Shamiri, School of Mathematical Sciences, University Kebangsaan Malaysia, 43600 UKM, Bangi, Malaysia 
Many authors do not observe anything special on this point, but other researchers, such as Simkowitz and Beedles $^{[13]}$ and $\mathrm{Kon}^{[7]}$ have drawn the attention to the heavy tail of the distribution. Shamiri ${ }^{[10,12]}$ have shown that a fat-tailed distribution is required for modeling daily returns of East Asian equity markets.

This study adds to the literature in several important directions. Firstly, in this study we demonstrate that this gap can be filled by a rigorous density forecast models comparison methodology. Secondly, we compare the performance of the GARCH, EGARCH and NAGARCH models and also introduce different densities (Normal, Skew normal, Student-t, Skew student-t, Generalized Error Distribution GED and Normal Inverse Gaussian (NIG)). Thirdly, using high-frequency data from Kuala Lumpur Composite Index (KLCI) of Malaysian's stock market to facilitate meaningful comparison of the forecast results. In the model estimation, it is shown that allowing for a leptokurtic return distribution significantly improves the fit of the model. In terms of out-of-sample forecasting performance, allowing for excess kurtosis leads to significant improvements over the normal distribution, whereas allowing for non-centrality does not further enhance forecasts.

\section{MATERIALS AND METHODS}

Data source: All data are the daily data obtained from DataStream. In the database, the daily return $R_{t}$ consisted of daily stock closing price $\mathrm{P}_{t}$ of KLCI, which is measured in local currency. The sample consists of 2869 daily observations on stock returns of the KLCI index. The index spans a period of approximately 11 years from $1 / 1 / 1998$ to $31 / 12 / 2008$. In the database, the daily return $R_{t}$ consisted of daily closing price $P_{t}$, which is measured in local currency and computed as:

$$
R_{t}=\ln \left[P_{t} / P_{t-1}\right]
$$

GARCH: Bollersle ${ }^{[3]}$,introduced GARCH model known as the Generalized auto-regressive conditional heteroskedasticity model which suggest that the timevarying volatility process is a function of both past disturbances and past volatility. The GARCH model is an infinite order ARCH model generated by:

$$
\sigma_{t}^{2}=\alpha_{0}+\sum_{i=1}^{p} \alpha_{i} \varepsilon_{t-i}^{2}+\sum_{j=1}^{q} \beta_{j} \sigma_{t-j}^{2}
$$

where $\alpha_{0}, \alpha$ and $\beta$ are non-negative constants. For the GARCH process to be defined, it is required that $\alpha>0$.
EGARCH: Nelson ${ }^{[8]}$ introduced the first asymmetric GARCH model known as exponential GARCH model (EGARCH). This model looks at the conditional variance and tries to accommodate for the asymmetric relation between stock returns and volatility changes. Nelson implements that by including an adjusting function $\mathrm{g}(\mathrm{z})$ in the conditional variance equation, it in turn becomes expressed by:

$\ln \sigma_{t}^{2}=\alpha_{0}+\sum_{i=1}^{p} \alpha_{i} g\left(z_{t-i}\right)+\sum_{j=1}^{q} \beta_{j} \ln \left(\sigma_{t-j}^{2}\right)$

where, $z_{t}=\varepsilon_{t} / \sigma_{t}$ is the normalized residual series. The value of $g\left(z_{t}\right)$ is a function of both the magnitude and sign of $z_{t}$ and is expressed as:

$$
\mathrm{g}\left(\mathrm{z}_{\mathrm{t}}\right)=\underbrace{\theta_{1} \mathrm{z}_{\mathrm{t}}}_{\text {sign effect }}+\underbrace{\theta_{2}\left[\left|z_{\mathrm{t}}\right|-\mathrm{E}\left|\mathrm{z}_{\mathrm{t}}\right|\right]}_{\text {magnitude effect }}
$$

Notice moreover that $E\left|z_{t}\right|$ depends on the assumption made on the unconditional density. The EGARCH model differs from the standard GARCH model in two main aspects. First, it allows positive and negative shocks to have a different impact on volatility. Second, the EGARCH model allows large shocks to have a greater impact on volatility than the standard GARCH model.

NAGARCH: Engle $^{[5]}$ proposed the Asymmetric NonLinear GARCH Model (NAGARCH), given by:

$$
\sigma_{t}^{2}=\alpha_{0}+\sum_{i=1}^{p} \alpha_{i}\left(\varepsilon_{t-1}+\lambda \sigma_{t-1}^{2}\right)^{2}+\sum_{j=1}^{q} \beta_{j} \sigma_{t-1}^{2}
$$

Failure to capture fat-tails property of highfrequency financial time series has led to the use of non-normal distributions to better model excessive third and fourth moments. Since it may be expected that excess kurtosis and skewness displayed by the residuals of conditional heteroskedasticity models will be reduced when a more appropriate distribution is used, we consider six distributions in this study: the normal, skew-normal, student-t, skew-student-t, GED and NIG. In the following, $f(z)$ is the standardized density function of the standardized residuals $\left\{z_{t}\right\}$.

\section{Normal distribution:}

$f(z)=\frac{1}{\sqrt{2 \pi}} \exp \left(-\frac{z^{2}}{2}\right), E|z|=\sqrt{2 / \pi}, \gamma_{2}=0$ 
Skew-normal distribution:

$$
\begin{aligned}
& \mathrm{f}(\mathrm{z})=\frac{1}{\sigma \pi} \mathrm{e}^{-\frac{(\mathrm{z}-\zeta)^{2}}{\sigma}} \int_{-\infty}^{\alpha\left(\frac{\mathrm{z}-\zeta}{\sigma}\right)} \mathrm{e}^{-\frac{\mathrm{t}^{2}}{2}} \mathrm{dt} \\
& \mathrm{E}|\mathrm{z}|=\zeta+\sigma \delta \sqrt{\frac{2}{\pi}} \\
& \gamma_{1}=\frac{4-\pi}{2} \frac{(\delta \sqrt{2 / \pi})^{3}}{\left(1-2 \delta^{2} / \pi\right)^{3 / 2}}, \\
& \gamma_{2}=2(\pi-3) \frac{(\delta \sqrt{2 / \pi})^{4}}{\left(1-2 \delta^{2} / \pi\right)^{2}}
\end{aligned}
$$

$\xi$ and $\alpha$ are the location scale and shape respectively, where, $\delta=\frac{\alpha}{\sqrt{1+\alpha^{2}}}$.

\section{Student-t distribution:}

$$
\begin{aligned}
& f(\mathrm{z} ; \mathrm{v})=\frac{\Gamma(\mathrm{v}+1 / 2)}{\Gamma(\mathrm{v} / 2)} \frac{1}{\sqrt{\pi(\mathrm{v}-2)}}\left(1+\frac{\mathrm{z}^{2}}{\mathrm{v}-2}\right)^{-(\mathrm{v}+1 / 2)}, \mathrm{v}>2 \\
& \mathrm{E}|\mathrm{z}|=\frac{4 \Gamma(1+\mathrm{v} / 2) \sqrt{\mathrm{v}-2}}{1+\sqrt{\pi} \Gamma(\mathrm{v} / 2)(\mathrm{v}-1)} \\
& \gamma_{2} \quad=\frac{6}{\mathrm{v}-4}, \mathrm{v}>4
\end{aligned}
$$

\section{Skewed-t distribution:}

$$
\begin{aligned}
& \mathrm{f}(\mathrm{z})=\left\{\begin{array}{l}
\left(2 \mathrm{~s} / \zeta+\zeta^{-1}\right)[\Gamma(\mathrm{v}+1 / 2) / \Gamma(\mathrm{v} / 2)](1 / \sqrt{\pi(\mathrm{v}-2)}) \\
{\left[1+\zeta^{2}(\mathrm{sz}+\mathrm{m})^{2} / \mathrm{v}-2\right]^{-(\mathrm{v}+1 / 2)}} \\
\left(2 \mathrm{~s} / \zeta+\zeta^{-1}\right)[\Gamma(\mathrm{v}+1 / 2) / \Gamma(\mathrm{v} / 2)](1 / \sqrt{\pi(\mathrm{v}-2)}) \\
{\left[1+\zeta^{-2}(\mathrm{sz}+\mathrm{m})^{2} / \mathrm{v}-2\right]^{-(\mathrm{v}+1 / 2)} \quad \text { if } \mathrm{z}>-\mathrm{m} / \mathrm{s}}
\end{array}\right. \\
& \mathrm{E}|\mathrm{z}|=\frac{\left.4 \zeta^{2} \Gamma(1+\mathrm{v}) / 2\right) \sqrt{\mathrm{v}-2}}{\zeta+1 / \zeta \sqrt{\pi} \Gamma(\mathrm{v} / 2)(\mathrm{v}-1)} \\
& \gamma_{1}=\frac{(\mathrm{v}-2)^{3 / 2}\left(\zeta^{2}-1\right)\left(\zeta^{4}+1\right) \Gamma[(\mathrm{v}-3) / 2]}{\sqrt{\pi} \zeta^{3} \mathrm{~s}^{3} \Gamma(\mathrm{v} / 2)}-\frac{\mathrm{m}^{2}\left(\mathrm{~m}^{2}+3 \mathrm{~s}\right)}{\mathrm{s}^{3}}( \\
& \gamma_{2}=\frac{3\left(\zeta^{5}+\zeta^{-5}\right)(\mathrm{v}-2)}{\left(\zeta+\zeta^{-1}\right)(\mathrm{v}-4) \mathrm{s}^{4}}-\frac{3 \mathrm{~m}^{2}\left(\mathrm{~m}^{2}+2 \mathrm{~s}\right)}{\mathrm{s}^{4}} \\
& -\frac{4 \mathrm{~m}(\mathrm{v}-2)^{3 / 2}\left(\zeta^{2}-1\right)\left(\zeta^{4}+1\right) \Gamma[(\mathrm{v}-3) / 2]}{\sqrt{\pi} \zeta^{3} \mathrm{~s}^{4} \Gamma(\mathrm{v} / 2)}
\end{aligned}
$$

where, $\quad \zeta>0, \mathrm{v}>2, \quad \mathrm{~m}=\Gamma[(\mathrm{v}-1) / 2] \sqrt{(\mathrm{v}-2) / \pi}(\zeta-1 / \zeta) /$ $\Gamma(\mathrm{v} / 2), \mathrm{s}=\sqrt{\left(\zeta^{2}+1 / \zeta^{2}-1\right)-\mathrm{m}^{2}} \quad \gamma_{1}$ exists if $\mathrm{v}>3$ and $\gamma_{2}$ exists if $v>4$.
GED:

$$
\begin{aligned}
\mathrm{f}(\mathrm{z} ; \mathrm{v}) & =\frac{\mathrm{vexp}\left(-0.5|\mathrm{z} / \lambda|^{\mathrm{v}}\right)}{\lambda 2^{(1+1 / \mathrm{v})} \Gamma(1 / \mathrm{v})} \\
\mathrm{v}>0, \lambda & =\sqrt{2^{(-2 / v)} \Gamma(1 / \mathrm{v}) / \Gamma(3 / \mathrm{v})} \\
\gamma_{2} & =\frac{\Gamma(1 / \mathrm{v}) \Gamma(5 / \mathrm{v})}{[\Gamma(3 / \mathrm{v})]^{2}}
\end{aligned}
$$

NIG:

$$
\begin{aligned}
& \mathrm{f}(\mathrm{z})=\left(\frac{\tau}{\pi}\right) \phi(\mathrm{z})^{-1 / 2} \mathrm{~K}_{1}(\tau \delta \sqrt{\phi(\mathrm{z})}) \\
& \exp \left(\delta \sqrt{\tau^{2}-\beta^{2}}+\beta(\mathrm{z}-\mu)\right) \\
& \mathrm{E}|\mathrm{z}|=\mu+\frac{\delta \beta}{\omega} \\
& \gamma_{1}= \frac{3 \beta}{\tau(\delta \omega)^{1 / 2}}, \\
& \gamma_{2}=3+\frac{3\left(1+4 \beta^{2} / \tau^{2}\right)}{\delta \omega}
\end{aligned}
$$

where, $\quad \tau=\sqrt{\omega^{2}+\beta^{2}}, \quad \phi(\mathrm{z})=1+((\mathrm{z}-\mu) / \delta)^{2}$, $\mathrm{K}(\mathrm{u})=\frac{1}{2} \int \mathrm{y}^{\lambda-1} \exp \left\{-\frac{\mathrm{u}}{2}\left(\mathrm{y}+\frac{1}{\mathrm{y}}\right)\right\} \mathrm{dy}, \mathrm{u}>0 \quad$ denotes the modified Bessel function of the third kind of order $\lambda$ evaluated at $u$ and $w=\sqrt{\tau^{2}-\beta^{2}}$ let $E_{i, T}\left(h_{T+h}\right)$ denote the $h$-step ahead forecast of $h_{T+h}$ at time T from GARCH model $\mathrm{i}$ using rolling methods. Define the corresponding forecast error as $\varepsilon_{\mathrm{I}, \mathrm{T}+\mathrm{h}}=\mathrm{E}_{\mathrm{i}, \mathrm{T}}\left(\mathrm{h}_{\mathrm{T}+\mathrm{h}}\right)-\mathrm{h}_{\mathrm{T}+\mathrm{h}}$ Common evaluation statistics based on Mean Squared Error (MSE), Mean Absolute Error (MAE), Median Squared Error (MDSE) and Root Mean Squared Error (RMSE).

\section{RESULTS}

Descriptive statistics: Table 1 shows the descriptive statistics of KLCI series for the sample under consideration. The mean return is positive $0.014 \%$, however, accompanied by high volatility $1.51 \%$. It is clear that the Malaysian market offer high average returns but these high returns are also characterized by high volatility, which is common for emerging markets and is consistent with previous studies ${ }^{[9,10]}$. Moreover, we check the statistical features of the data reported in Table 1, the skewness, kurtosis and their tests.

The Ljung-Box Q-statistics Q (10) and $\mathrm{Q}^{2}(10)$ are reported under the null hypothesis of non-serial correlation tests in daily return and squared returns, 
Table 1: Summary statistics for daily returns 1 January 1998-31 December 2008

\begin{tabular}{|c|c|c|c|c|c|c|c|c|c|c|}
\hline & Sample & Mean & Std. & Skewness & Kurtosis & Rob.Sk & Rob.Kr & $\mathrm{Q}(10)$ & $Q^{2}(10)$ & LM (5) \\
\hline KLCI & 2870 & 0.0135 & 1.506 & 0.569 & 60.239 & -0.004 & 0.37 & $85.29 * *$ & $1365 * *$ & $787 * *$ \\
\hline
\end{tabular}
presence of ARCH at lag 5, **,*: Significant at 1 and $5 \%$ respectively

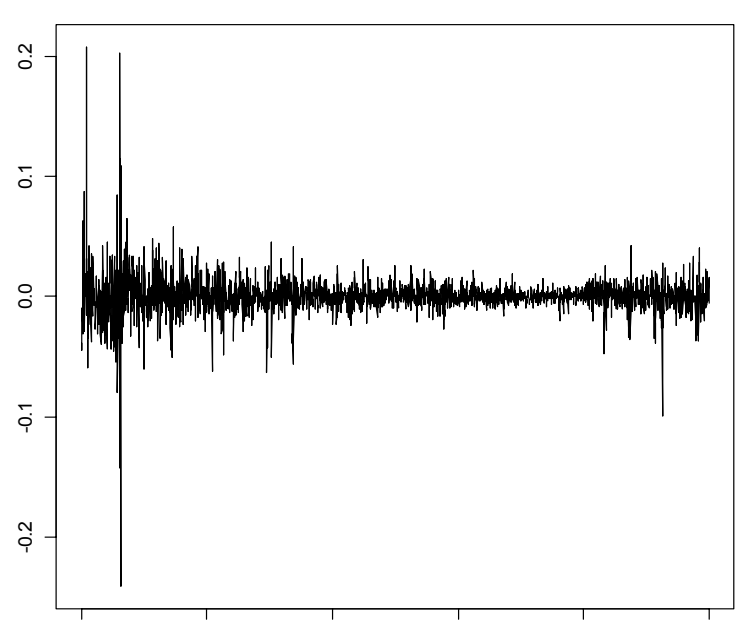

$1998-01-02 \quad 2000-03-16 \quad 2002-05-28 \quad 2004-08-09 \quad 2006-10-19 \quad 2008-12-31$

Fig. 1: KLCI daily returns

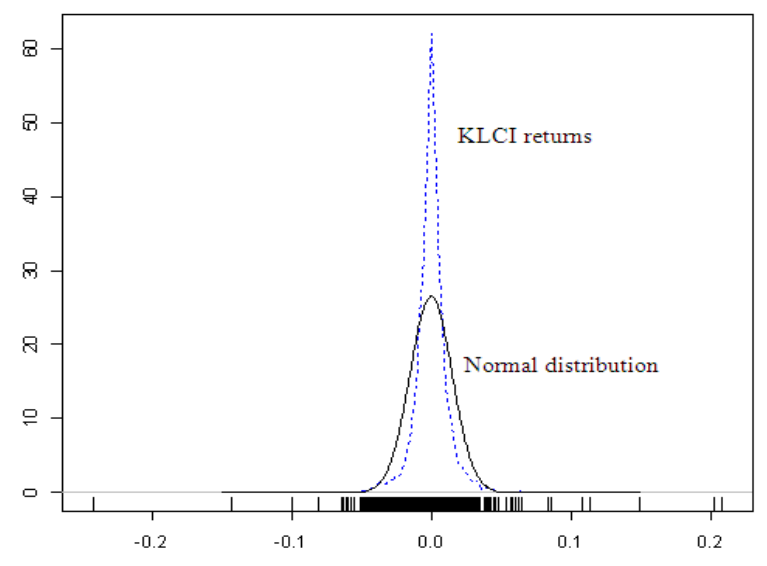

Fig. 2: KLCI daily return distribution

respectively. At significance levels of $5 \%$, the null hypotheses (skewness $=0$ or excess kurtosis $=0$ ) and of non-serial correlation are rejected, respectively. This time series have the typical features of stock returns as fat tail, spiked peak and persistence in variance. In contrast, the robustified statistics, Rob.Sk and Rob.Kr, do not suggest non-normality. With evidence of ARCH effects, it is possible to proceed to the second step of the analysis focused on the GARCH modeling of market's volatility.
Figure 1 looks at the behavior of the KLCI returns, over the sample period. There is evidence of volatility clustering and that large or small asset price changes tend to be followed by other large or small price changes of either sign (positive or negative). This implies that stock return volatility changes over time. Furthermore, the figure indicates that the Malaysian equity market is not affected much by US recent financial crisis compared to the Asian crisis in 1997. Figure 2 shows the distribution of KLCI log returns, which clearly indicate the departure from normality with a high peaked distribution.

Estimation and diagnostic: A quasi maximum likelihood approach is used to estimate the Models GARCH, EGARCH and NAGARCH, with six underlying error distributions. Table 2 , shows the estimation results for the parameters for the GARCH, EGARCH and NAGARCH models. The use of asymmetric GARCH models seems to be justified. All asymmetric coefficients are significant at standard levels. Moreover, the Akaike Information Criteria (henceforward AIC) and the Bayesian information criterion values highlight the fact that EGARCH and NAGARCH models better estimate the series than the traditional GARCH. All the models seem to do a good job in describing the dynamic of the first two moments of the series as shown by the Box-Pierce statistics for the squared standardized residuals with lag 15 which are all non-significant at 5\% level. LM test for presence of ARCH effects at lag 5, indicate that the conditional hetroskedasity that existed when the test was performed on the pure return series (Table 1) are removed. As, is typical of GARCH model estimates for financial asset returns data, the sum of the coefficients on the lagged squared error $\left(\alpha_{1}\right)$ and the lagged conditional variance $\left(\beta_{1}\right)$ is close to unity 1.00 and 0.99 with the GED and NIG error term respectively, this implies that shocks to the conditional variance well be highly persistent indicating that large changes and small changes tend to be followed by small changes, this mean volatility clustering is observed in KLCI financial returns series. 
J. Math. \& Stat., 5 (3):234-240, 2009

Table 2: GARCH models estimation

\begin{tabular}{|c|c|c|c|c|c|c|}
\hline & Normal & Skew normal & Student- $\mathrm{t}$ & Skew student & GED & NIG \\
\hline \multicolumn{7}{|c|}{ Panel A: GARCH: } \\
\hline \multirow[t]{2}{*}{$\alpha_{0}$} & $0.0000^{* *}$ & $0.0000 * *$ & $0.0000^{* *}$ & $0.0000^{* *}$ & $0.0000^{* *}$ & $0.0000 * *$ \\
\hline & $(0.0000)$ & $(0.0000)$ & $(0.00000)$ & $(0.00000)$ & $(0.0000)$ & $(0.0000)$ \\
\hline \multirow[t]{2}{*}{$\alpha_{1}$} & $0.1185^{* *}$ & $0.1177^{* *}$ & $0.14790 * *$ & $0.14850^{* *}$ & $0.1306^{* *}$ & $0.1312 * *$ \\
\hline & $(0.0111)$ & $(0.0111)$ & $(0.02180)$ & $(0.02190)$ & $(0.0189)$ & $(0.0183)$ \\
\hline \multirow[t]{2}{*}{$\beta_{1}$} & $0.8874 * *$ & $0.8881 * *$ & $0.86170^{* *}$ & $0.86150^{* *}$ & $0.8735^{* *}$ & $0.8686 * *$ \\
\hline & $(0.0092)$ & $(0.0093)$ & $(0.01710)$ & $(0.01710)$ & $(0.0159)$ & $(0.0160)$ \\
\hline \multirow[t]{2}{*}{$\gamma_{1}$} & & $1.0122 * *$ & & $0.98630 * *$ & & -0.0145 \\
\hline & & $(0.0197)$ & & $(0.02250)$ & & $(0.0178)$ \\
\hline \multirow[t]{2}{*}{$\gamma_{2}$} & & $4.0477 * *$ & $4.04160 * *$ & $1.02360 * *$ & $1.0000^{* *}$ & \\
\hline & & $(0.3345)$ & $(0.33420)$ & $(0.05580)$ & $(0.1463)$ & \\
\hline Log like & -9113.6290 & -9113.8230 & -9287.87900 & -9288.06000 & -9301.719 & -9290.2330 \\
\hline $\mathrm{AIC}$ & -6.3504 & -6.3498 & -6.47120 & -6.47060 & -6.4808 & -6.47210 \\
\hline BIC & -6.3421 & -6.3394 & -6.46080 & -6.45810 & -6.4704 & -6.45960 \\
\hline Q2(15) & 8.4750 & 8.4800 & 7.64900 & 7.65500 & 7.786 & 7.72000 \\
\hline $\operatorname{LM}(5)$ & 2.2450 & 2.2770 & 1.65710 & 1.65970 & 1.7187 & 1.66470 \\
\hline \multicolumn{7}{|c|}{ Panel B: EGARCH: } \\
\hline \multirow[t]{2}{*}{$\alpha_{0}$} & $-0.0682 * *$ & $-0.0697 * *$ & $-0.19600 * *$ & $-0.19850 * *$ & $-0.1451 * *$ & $-0.1717 * *$ \\
\hline & $(0.0195)$ & $(0.0201)$ & $(0.04680)$ & $(0.04790)$ & $(0.0372)$ & $(0.0412)$ \\
\hline \multirow[t]{2}{*}{$\alpha_{1}$} & 0.2417 & 0.4144 & 0.78050 & 0.48790 & 0.4076 & 0.37670 \\
\hline & $(0.4169)$ & $(0.5892)$ & $(0.95070)$ & $(0.43130)$ & $(0.5362)$ & $(0.49570)$ \\
\hline \multirow[t]{2}{*}{$\beta_{1}$} & $0.9911 * *$ & $0.9910 * *$ & $0.97860 * *$ & $0.97830 * *$ & $0.9843^{* *}$ & $0.981500^{* *}$ \\
\hline & $(0.0021)$ & $(0.0022)$ & $(0.00510)$ & $(0.00520)$ & $(0.0040)$ & $(0.00450)$ \\
\hline \multirow[t]{2}{*}{$\phi_{1}$} & $-0.0808^{*}$ & $-0.1290 * *$ & $-0.08660 * *$ & $-0.13830 * *$ & $-0.1399 * *$ & $-0.16660 * *$ \\
\hline & $(0.0036)$ & $(0.0027)$ & $(0.00180)$ & $(0.00130)$ & $(0.0049)$ & $(0.00540)$ \\
\hline \multirow[t]{2}{*}{$\phi_{2}$} & $0.3003 *$ & $0.4705^{* *}$ & $0.32060 * *$ & $0.52040 * *$ & $0.5343^{* *}$ & $0.61810 * *$ \\
\hline & $(0.0776)$ & $(0.0104)$ & $(0.09270)$ & $(0.01810)$ & $(0.0048)$ & $(0.01270)$ \\
\hline \multirow[t]{2}{*}{$\gamma_{1}$} & & $0.9766^{* *}$ & & $0.96360 * *$ & & -0.05550 \\
\hline & & $(0.0192)$ & & $(0.02310)$ & & $(0.04270)$ \\
\hline \multirow[t]{2}{*}{$\gamma_{2}$} & & & $4.22540 * *$ & $4.19210 * *$ & $1.0786^{* *}$ & $1.00000 * *$ \\
\hline & & & $(0.35380)$ & $(0.34980)$ & $(0.0376)$ & $(0.13650)$ \\
\hline Log like & -9155.3020 & -9156.0020 & -9330.24800 & -9330.62000 & -9328.751 & -9330.03200 \\
\hline AIC & -6.3773 & -6.3771 & -6.49790 & -6.49820 & -6.4976 & -6.49780 \\
\hline $\mathrm{BIC}$ & -6.3628 & -6.3605 & -6.47920 & -6.47950 & -6.4809 & -6.47910 \\
\hline Q2(15) & 9.7570 & 9.4220 & 5.09100 & 4.06400 & 5.773 & 4.69100 \\
\hline $\mathrm{LM}(5)$ & 5.6840 & 5.3950 & 0.88110 & 0.88780 & 2.166 & 1.33330 \\
\hline \multicolumn{7}{|c|}{ Panel C: NAGARCH: } \\
\hline \multirow[t]{2}{*}{$\alpha_{0}$} & $0.0000 * *$ & $0.0000^{* *}$ & $0.00000 * *$ & $0.00000 * *$ & $0.0000^{* *}$ & $0.0000 * *$ \\
\hline & $(0.0000)$ & $(0.0000)$ & $(0.00000)$ & $(0.00000)$ & $(0.0000)$ & $(0.0000)$ \\
\hline \multirow[t]{2}{*}{$\alpha_{1}$} & $0.1124 * *$ & $0.1132 * *$ & $0.14520 * *$ & $0.14600^{* *}$ & $0.1287^{* *}$ & $0.13070^{* *}$ \\
\hline & $(0.0106)$ & $(0.0108)$ & $(0.02150)$ & $(0.02140)$ & $(0.0183)$ & $(0.01810)$ \\
\hline \multirow[t]{2}{*}{$\beta_{1}$} & $0.8770 * *$ & $0.8757 * *$ & $0.84770 * *$ & $0.84580 * *$ & $0.8588 * *$ & $0.85200 * *$ \\
\hline & $(0.0099)$ & $(0.0103)$ & $(0.01870)$ & $(0.01870)$ & $(0.0173)$ & $(0.01780)$ \\
\hline$\gamma$ & $0.3428 * *$ & $0.3475^{* *}$ & $0.31260 * *$ & $0.32190 * *$ & $0.3503 * *$ & $0.346700 * *$ \\
\hline & $(0.0515)$ & $(0.0523)$ & $(0.06470)$ & $(0.06570)$ & $(0.0696)$ & $(0.06880)$ \\
\hline$\gamma_{1}$ & & $0.9873 * *$ & & $0.97150 * *$ & & -0.04660 \\
\hline & & $(0.0195)$ & & $(0.02260)$ & & $(0.03540)$ \\
\hline$\gamma_{2}$ & & & $4.11600 * *$ & $4.11280^{* *}$ & $1.0341 * *$ & $1.000000^{* *}$ \\
\hline & & & $(0.34350)$ & $(0.34420)$ & $(0.0407)$ & $(0.14470)$ \\
\hline Log like & -9137.3100 & -9137.5190 & -9300.47800 & -9301.25700 & -9315.2340 & -9304.15000 \\
\hline $\mathrm{AIC}$ & -6.3662 & -6.3656 & -6.47920 & -6.47910 & -6.4895 & -6.48110 \\
\hline $\mathrm{BIC}$ & -6.3558 & -6.3532 & -6.46680 & -6.46450 & -6.4771 & -6.46660 \\
\hline Q2(15) & 8.8690 & 8.8380 & 8.51200 & 8.46600 & 8.5310 & 8.43100 \\
\hline $\operatorname{LM}(5)$ & 2.6150 & 2.5790 & 2.22845 & 2.22186 & 2.1743 & 2.14490 \\
\hline
\end{tabular}

Standard errors are given in parentheses. **,*: Significant at 1 and $5 \%$ respectively

DISCUSSION

Based on the findings, the symmetric distributions with fatter tails clearly outperform the Gaussian. According to AIC, GARCH and NAGARCH models perform better with GED, while EGARCH model perform better with skew-student-t distribution. The leverage effect terms $\phi_{1}$ and $\phi_{2}$ in EGARCH model and asymmetric term $\lambda$ in NAGARCH model are statistically significant, furthermore with $\phi_{1}$ negative sign, 
J. Math. \& Stat., 5 (3):234-240, 2009

Table 3: Forecast performance out-of- sample

\begin{tabular}{|c|c|c|c|c|c|c|}
\hline & Normal & Skew normal & Student- $\mathrm{t}$ & Skew student & GED & NIG \\
\hline \multicolumn{7}{|c|}{ Panel A: GARCH: } \\
\hline MSE & 0.001098 & 0.001098 & 0.001294 & 0.001313 & 0.001099 & 0.001080 \\
\hline MDSE & 0.000212 & 0.000212 & 0.000351 & 0.000362 & 0.000219 & 0.000159 \\
\hline RMSE & 3.313204 & 3.313204 & 3.597198 & 3.623870 & 3.314653 & 3.286948 \\
\hline MAE & 2.143484 & 2.143484 & 2.649073 & 2.683303 & 2.155249 & 2.008332 \\
\hline \multicolumn{7}{|c|}{ Panel B: EGARCH: } \\
\hline MSE & 0.001092 & 0.001072 & 0.001090 & 0.001090 & 0.001096 & 0.001100 \\
\hline MDSE & 0.000339 & 0.000339 & 0.000118 & 0.000115 & 0.000107 & 0.000100 \\
\hline RMSE & 3.304849 & 3.274849 & 3.300770 & 3.301175 & 3.309850 & 3.316831 \\
\hline MAE & 2.244085 & 2.244085 & 1.851566 & 1.852097 & 1.838961 & 1.833950 \\
\hline \multicolumn{7}{|c|}{ Panel C: NAGARCH: } \\
\hline MSE & 0.002087 & 0.002087 & 0.013221 & 0.011741 & 0.003112 & 0.001978 \\
\hline MDSE & 0.000908 & 0.000908 & 0.003131 & 0.002976 & 0.001271 & 0.000850 \\
\hline RMSE & 4.568193 & 4.568193 & 11.498303 & 10.835615 & 5.578780 & 4.447064 \\
\hline MAE & 3.645259 & 3.645259 & 8.491675 & 8.080493 & 4.479974 & 3.552767 \\
\hline
\end{tabular}

MSE: Mean squared error; MDSE: The median squared error; RMSE: The root mean squared error; MAE: The mean absolute error

as expected that negative shocks imply a higher next period conditional variance than positive shocks of the same sign, indicating that the existence of leverage effect is observed in returns of the KLCI market index. However, the comparison between models with each density (normal versus non-normal) shows that, according to the different measures used for modeling the volatility, the EGARCH model with skew-student- $\mathrm{t}$ provides the best in-sample estimation for KLCI compared to all other volatility models and distributions.

Now that we have estimated the series, the obvious question is how good are the forecasting models? Typically, there are several plausible models that we can select to use for our forecast. We should not be fooled into thinking that the one with the best fit is the one that will forecast the best.

The results of forecasting daily volatility with GARCH models together with various distributions and four evaluation criteria are given in Table 3 . All results are presented for each distribution and for each model GARCH models, whose specification is always of order $(1,1)$. In this study the length of the out-of-sample period is chosen to be 100 days. Form Table 3 some interesting comments emerge. A first major conclusion is that there is no single model that completely dominates the other models. Secondly, forecasting with normal distribution does not yield a significant reduction of the forecast error relative to heavy-tailed distributions. Thus, the failure of predictor $h_{T+h}$ is justified due to that the GARCH model residuals follow a (possibly) heavy-tailed distribution. Third, it seems that asymmetric model (EGARCH) tend to perform better forecast with a fatter tailed distribution (student- $t$ and Skew-student-t). It is apparent, that the simple predictor $h_{T+h}$ seems to actually have some predictive ability, when a heavy-tailed is assumed for the GARCH residuals (i.e., NIG distribution). A possible explanation is that modeling asymmetries contributes to the reduction of the magnitude of the bias.

\section{CONCLUSION}

This study contributes to the literature of volatility modeling in two aspects. First, we use a data set from an emerging market. Secondly we estimate the alternative GARCH-type models (symmetric and asymmetric GARCH Models). The comparison was focused on two different aspects: The difference between symmetric and asymmetric GARCH (i.e., GARCH versus EGARCH and NAGARCH) and the difference between normal tailed symmetric, heavytailed symmetric distributions and both heavy-tailed and asymmetric distributions for estimating the KLCI stock market index return volatility. As expected, the leverage KLCI market shown by EGARCH model is statistically significant at with a negative sign, which indicate that negative shocks imply a higher next period conditional variance than positive shocks of the same sign, indicating that the existence of leverage effect is observed in returns of the KLCI stock market index.

However, the comparison between models with each density (normal versus non-normal) shows that, according to the different measures used for the performance of volatility forecast, the EGARCH model provides the best out-sample estimation for KLCI clearly outperform the symmetric models. Our results show that, non-normal distributions provide better insample results than the normal distribution. Out-ofsample results show however less evidence of superior forecasting ability. Briefly, looking at the overall results, we can argue that the asymmetric model coupled with a Student-t distribution for the innovations performs very well with the dataset we have 
investigated. The models seems to capture the dynamics of the first and second moments of the KLCI stock market index returns series.

\section{REFERENCES}

1. Anders, W., 2006. Garch forecasting performance under different distribution assumptions. J. Forecast., $\quad 25: \quad 561-578$. http://direct.bl.uk/bld/PlaceOrder.do?UIN=200371 $640 \& \mathrm{ETOC}=\mathrm{RN} \&$ from $=$ searchengine

2. Andersen, T.G. and T. Bollerslev, 1998. Answering the skeptics: Yes standard variance models do provide accurate forecasts. Intl. Econ. Rev., 39: 885-905.

http://72.14.235.132/search?q=cache:gIqwCXka8A oJ:fmg.lse.ac.uk/upload_file/20_andersen.pdf + Ans wering+the+skeptics: $+\bar{Y}$ es + standard + variance + mo dels + do + provide + accurate + forecasts $\& c d=1 \& \mathrm{hl}=$ en $\& \mathrm{ct}=\mathrm{clnk} \& \mathrm{gl}=\mathrm{pk}$

3. Bollerslev, T., 1986. Generalized autoregressive conditional heteroskedasticity. J. Econ., 31: 307-327. http://econpapers.repec.org/article/eeeeconom/v_3 A31_3Ay_3A1986_3Ai_3A3_3Ap_3A307327.htm

4. Engle, R.F., 1982. Autoregressive conditional heteroscedasticity with estimates of the variance of United Kingdom inflation. Econometrica, 50: 987-1006. http://ideas.repec.org/a/ecm/emetrp/v50y1982i4p9 87-1007.html

5. Engle, R.F., 1990. Stock volatility and the crash of '87: Discussion. Rev. Financ., 3:103-106. http://rfs.oxfordjournals.org/cgi/content/citation/3/ $1 / 103$

6. Kim, T. and H. White, 2004. On more robust estimation of skewness and kurtosis: Simulation and application to the S and P500 index. Finance Res. Lett., 1: 56-73.

http://ideas.repec.org/a/eee/finlet/v1y2004ilp5673.html

7. Kon, S.J., 1984. Models of stock returns: A comparison. J. Finance, 39: 147-165. http://72.14.235.132/search?q=cache:gVJGXS9nG YMJ:efinance.org.cn/cn/fe/19840301Models\%252 0of\%2520Stock\%2520Returns-A\%2520Comparison,\%2520pp.\%2520147165.pdf+Models + of + stock+returns: + A+compariso $\mathrm{n} \& \mathrm{~cd}=3 \& \mathrm{hl}=\mathrm{en} \& \mathrm{ct}=\mathrm{clnk} \& \mathrm{gl}=\mathrm{pk}$
8. Nelson, D.B., 1991. Conditional heteroskedasticity in asset returns: A new approach. Econometrica, 59: 347-370.

http://econpapers.repec.org/article/ecmemetrp/v_3a 59_3ay_3a1991_3ai_3a2_3ap_3a347-70.htm

9. $\mathrm{Ng}$, A., 2000. Volatility spillover effects from Japan and the US to the Pacific-Basin. J. Int. Money Finance, 19: 207-233. http://econpapers.repec.org/article/eeejimfin/v_3a1 9_3ay_3a2000_3ai_3a2_3ap_3a207-233.htm

10. Shamiri, A. and M.S.N. Abu Hassan, 2007. Modelling and forecasting volatility of the Malaysian and Singaporean stock indices using asymmetric GARCH models and non-normal densities. J. Malaysian Math. Sci., 1: 83-102. http://ideas.repec.org/p/wpa/wuwpem/0509015.html

11. Shamiri, A., M.S.N. Abu Hassan and I. Zaidi, 2005. Practical volatility modeling for financial market risk management. Working Paper 9790 MPRA. http://ideas.repec.org/p/pra/mprapa/9790.html

12. Shamiri, A., M.S.N. Abu Hassan and I. Zaidi, 2009. Comparing the accuracy of density forecasts from competing GARCH models. Sains Malaysiana, 38: 109-118. http://ideas.repec.org/p/pra/mprapa/13662.html

13. Simkowitz, M.A. and W.L. Beedles, 1980. Asymmetric stable distributed security returns. J. Am. Stat. Assoc., 75: 306-312. http://www.jstor.org/pss/2287449

14. Taylor, S., 1986. Modelling Financial Time Series. Wiley Chichester Publisher, ISBN: 0471909939, pp: 268. 